\title{
Pacific
}

Journal of

Mathematics

\section{ON THE ODD PRIMARY COHOMOLOGY OF HIGHER PROJECTIVE PLANES}

\author{
Mark Foskey AND Michael David Slack
}




\title{
ON THE ODD PRIMARY COHOMOLOGY OF HIGHER PROJECTIVE PLANES
}

\author{
Mark Foskey and Michael Slack
}

\begin{abstract}
Let $X$ be an $n$-fold loop space. Working with an auxiliary space $P_{p}^{n} X$ analogous to the projective plane $P_{2} X$, we show that the existence of certain Steenrod connections in $H^{*}\left(P_{p}^{n} X ; \mathbf{F}_{p}\right)$ ( $p$ odd $)$ implies the vanishing of certain corresponding Dyer-Lashof operations in $H^{*}\left(X ; \mathbf{F}_{p}\right)$, and vice versa.
\end{abstract}

\section{Introduction.}

A useful property of the projective plane of an H-space is that the vanishing of certain cup products in $H^{*}\left(P_{2} X\right)$ implies the existance of certain related nonzero reduced coproducts in $H^{*}(X)$ (see [BT]). In [KSW], Kuhn, Slack, and Williams introduce the necessary theory to construct a space $P_{p}^{n} X$ with the property that the Steenrod action on its cohomology bears a similar relation to the Dyer-Lashof action on the homology of $X$. We develop this relation at odd primes in this paper. In particular, our main theorem can be summarized as follows:

Main Theorem. Suppose $X$ is an $n$-fold loop space, $1 \leq n \leq \infty$. Then there is a certain cofibration sequence

$$
\Sigma^{n} \widetilde{C}_{n, p} X \stackrel{h}{\longrightarrow} \Sigma^{n} X \stackrel{i}{\longrightarrow} P_{p}^{n} X \stackrel{j}{\longrightarrow} \Sigma^{n+1} \widetilde{C}_{n, p} X
$$

such that

1. For each $x \in \widetilde{H}^{q}(X)$ there exist elements $\widetilde{Q}_{r} x \in \widetilde{H}^{p q+r}\left(\widetilde{C}_{n, p} X\right)$ such that $Q_{r}^{*} w=x$ whenever $h^{*}\left(\sigma^{n} w\right)=\sigma^{n} \widetilde{Q}_{r} x$, where $\sigma$ is the suspension isomorphism and

$$
Q_{r}^{*}: \widetilde{H}^{p q+r}(X) \rightarrow H^{q}(X)
$$

is an element of the opposite algebra to the Dyer-Lashof algebra.

2. If $\bar{x} \in \widetilde{H}^{2 s+1}\left(P_{p}^{n} X\right)$ and $i^{*}(\bar{x})=\sigma^{n} x$ then

$$
\begin{aligned}
\mathcal{P}^{s} \bar{x} & =u \cdot j^{*}\left(\sigma^{n+1} \widetilde{Q}_{(n-1)(p-1)-1} x\right), \\
\beta \mathcal{P}^{s} \bar{x} & =u \cdot j^{*}\left(\sigma^{n+1} \widetilde{Q}_{(n-1)(p-1)} x\right),
\end{aligned}
$$

where $u$ is some undetermined unit in $\mathbf{F}_{p}$. 
The analogous result at the prime 2 was proved in [KSW].

Using this theorem and the long exact sequence of a cofibration it is easy to see how the triviality of certain Steenrod operations in $\widetilde{H}^{*}\left(P_{p}^{n} X\right)$ can imply the existence of nontrivial Dyer-Lashof operations in $\widetilde{H}_{*}(X)$, and vice versa. In $[\mathbf{S l}]$, the second author exploits this relation (in the stable case, see Corollary 4.2) to prove the following result.

Theorem (Slack). If $X$ is a connected infinite loop space (of finite type), and all of the Dyer-Lashof operations are trivial on the mod $p$ homology of $X$, then $X$ is mod $p$ homotopy equivalent to a product of Eilenberg-MacLane spaces.

The definitions of the spaces $P_{p}^{n} X$ and $\widetilde{C}_{n, p} X$ are briefly summarized in Section 2. In Section 3 we define the elements $\widetilde{Q}_{r} x$ and show how they relate to the homology Dyer-Lashof operations, and in Section 4 we prove Part 2 of the Main Theorem. We conclude with an appendix giving the Nishida relations as they apply to the external operations $\widetilde{Q}_{r}$, which are useful in applications.

We summarize here some of the notational conventions used in this paper. Let $p$ be an odd prime, and take all homology and cohomology with coefficients in the field $\mathbf{F}_{p}$ of $p$ elements. Recall ([CLM]) that the homology of an $n$-fold loop space $X$ admits certain Dyer-Lashof operations $Q_{r}: \widetilde{H}_{q}(X) \rightarrow \widetilde{H}_{p q+r}(X)$. (We are using the lower notation of [CPS] in which $Q_{s(p-1)} v$ is defined to be $Q^{(s+\operatorname{deg} v) / 2} v$.) In this paper, all spaces will be compactly generated Hausdorff spaces with non-degenerate basepoint $*$, and all maps will be based maps.

We would like to thank Nicholas Kuhn, Jim Lin, and Fred Cohen for many helpful notes and conversations.

\section{The construction of the projective planes.}

In this section we recall relevant information from [KSW]. Let $C_{n} X$ denote the standard approximation to $\Omega^{n} \Sigma^{n} X$ of [Ma2, Mi]. It admits a filtration

$$
X \simeq C_{n, 1} X \subset C_{n, 2} X \subset \cdots \subset C_{n} X .
$$

If there is a map $\theta_{n}^{X}: C_{n, p} X \rightarrow X$ extending the identity on $X$, then $X$ is called an $H_{p}^{n}$-space, and there is a category $\mathcal{H}_{p}^{n}$ for which the objects are $H_{p}^{n}$ spaces and the morphisms can be thought of as homotopy classes of maps preserving the $H_{p}^{n}$-structure. ${ }^{1}$ From now on $X$ will generally be assumed

${ }^{1}$ Actually, they only preserve the $H_{p}^{n}$-structure up to homotopy, but they come equipped with prescribed classes of homotopies making the appropriate diagram commute. 
to be an $H_{p}^{n}$-space, and, in the main theorem, the hypothesis that $X$ is an $n$-fold loop space may be replaced by the somewhat weaker hypothesis that $X \in \mathrm{Ob} \mathcal{H}_{p}^{n}$.

There is a functor $\Omega^{n}: \mathcal{H}_{p}^{0} \rightarrow \mathcal{H}_{p}^{n}$ that, on objects, is the usual $n$-fold loop space functor. It admits a left adjoint $P_{p}^{n}: \mathcal{H}_{p}^{n} \rightarrow \mathcal{H}_{p}^{0}$ that is constructed (on objects) as follows. If $X$ is an $H_{p}^{n}$-space, there are two natural retractions $\Sigma^{n} C_{n, p} X \rightarrow \Sigma^{n} X$; one is $\Sigma^{n} \theta_{n}^{X}$ and the other is the adjoint $\varepsilon_{n}$ of the composition

$$
C_{n, p} X \hookrightarrow C_{n} X \longrightarrow \Omega^{n} \Sigma^{n} X
$$

If $\widetilde{C}_{n, p} X$ is the cofiber of the inclusion $X \hookrightarrow C_{n, p} X$, then there is a map $h: \Sigma^{n} \widetilde{C}_{n, p} X \rightarrow \Sigma^{n} X$, making the diagram

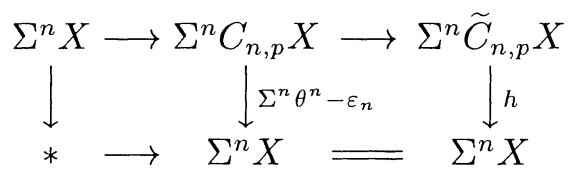

homotopy commute; $h$ is unique up to homotopy by the cofiber mapping sequence. The higher projective plane $P_{p}^{n} X$ is defined to be the cofiber of $h$, and we thus have the cofibration sequence

$$
\Sigma^{n} \widetilde{C}_{n, p} X \stackrel{h}{\longrightarrow} \Sigma^{n} X \stackrel{\imath}{\longrightarrow} P_{p}^{n} X \stackrel{j}{\longrightarrow} \Sigma^{n+1} \widetilde{C}_{n, p} X
$$

of the Main Theorem.

By way of comparison, the ordinary projective plane $P_{2} X$ can be defined as the cofiber of the map $h$ determined by the commutative diagram

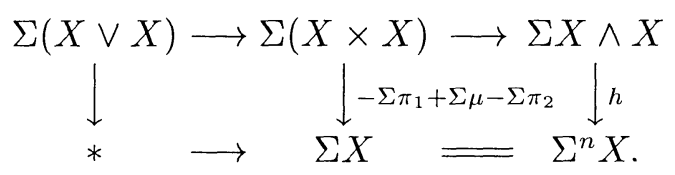

\section{The external operations.}

We now start to define the external operations $\widetilde{Q}_{r}$. We will begin by using the calculation of $H_{*}\left(C_{n} X\right)$ in $[\mathbf{C L M}]$ to observe that $H_{*}\left(\widetilde{C}_{n, p} X\right)$ can be viewed as a particular direct summand of $H_{*}\left(C_{n} X\right)$. We then use this information to produce a specific basis for $H_{*}\left(\widetilde{C}_{\infty, p} K(\mathbf{Z} / p, s+q-\gamma)\right)$, in terms of which we define a map

$$
G: \widetilde{C}_{\infty, p} K(\mathbf{Z} / p, s+q-\gamma) \longrightarrow K(\mathbf{Z} / p, p(s+q)-2 \gamma)
$$

(The roles of $s, q$, and $\gamma$, will be explained shortly.) Then, after recalling another map

$$
\tilde{\varepsilon}_{k}: \Sigma^{k} \widetilde{C}_{n+k, p} X \rightarrow \widetilde{C}_{n, p} \Sigma^{k} X,
$$


we define the external operations (Definition 3.3) in terms of $\tilde{\varepsilon}_{k}, G$, and a map $f$ representing $x \in H^{*}(X)$. We end the section by demonstrating some of their properties.

In this section, $r$ will denote $s(p-1)-\gamma$, where $\gamma \in\{0,1\}$, so that $Q_{r}: \widetilde{H}_{q}(X) \rightarrow \widetilde{H}_{p q+r}(X)$ will be defined on an $H_{p}^{n}$-space provided $s<n$ and $q+s$ is even. Recall ([CLM, III]) that, along with the Dyer-Lashof operations $Q_{r}$, the homology of $C_{n} X$ admits Browder operations

$$
\lambda_{n-1}: \widetilde{H}_{q}\left(C_{n} X\right) \otimes \widetilde{H}_{m}\left(C_{n} X\right) \longrightarrow \widetilde{H}_{q+m+n-1}\left(C_{n} X\right) .
$$

We will say that a Browder product of weight $l$ is a composition of Browder operations in which $l$ (not necessarily distinct) variables appear; for instance, $\lambda_{n-1}\left(x, \lambda_{n-1}(x, y)\right)$ is a Browder product of weight 3 . We recall that the Browder operations are neither associative nor commutative.

Let $\eta=\eta_{1}: X \rightarrow C_{n} X$ and $\eta_{k}: C_{n, k} X \rightarrow C_{n} X$ be the respective inclusions. We then have the following proposition, which summarizes information contained in Cohen's proof of the structure theorem for $\widetilde{H}_{*}\left(C_{n} X\right)$ ([CLM, III, Theorem 3.1]).

Proposition 3.1. The map $\left(\eta_{k}\right)_{*}: \widetilde{H}_{*}\left(C_{n, k} X\right) \rightarrow \widetilde{H}_{*}\left(C_{n} X\right)$ is one-to-one, and the filtration on homology defined by $F_{k} \widetilde{H}_{*}\left(C_{n} X\right)=\operatorname{im}\left(\eta_{k}\right)_{*}$ is identical to the following algebraic filtration: If $\lambda$ is a Browder product of weight $l$ applied to elements of $\eta_{*}\left(\widetilde{H}_{*}(X)\right)$, then the filtration of $Q_{r_{1}} \cdots Q_{r_{t}} \lambda$ is $p^{t} l$, and, if $a \in F_{j} \widetilde{H}_{*}\left(C_{n} X\right)$ and $b \in F_{k} \widetilde{H}_{*}\left(C_{n} X\right)$ then $a * b \in F_{j+k} \widetilde{H}_{*}\left(C_{n} X\right)$, where $a * b$ is the Pontryagin product of $a$ and $b$.

From this, along with the structure theorem for $\widetilde{H}_{*}\left(C_{n} X\right)$, it follows that $\operatorname{im}\left(\eta_{p}\right)_{*} \subset \widetilde{H}_{*}\left(C_{n} X\right)$ can be written as $A \oplus B \oplus C$, where $A, B$, and $C$ are defined as follows.

$$
\begin{aligned}
& A=\eta_{*} \widetilde{H}_{*}(X), \\
& B=\left\{Q_{s(p-1)-\gamma} \eta_{*}(x) \mid s>0,(s+\operatorname{deg} x) \text { is even }\right\}
\end{aligned}
$$

and $C$ is the set $\left\{\eta_{*}\left(x_{1}\right) * \cdots * \eta_{*}\left(x_{k}\right) * \lambda_{1} * \cdots * \lambda_{t}\right\}$, where $x_{1}, \ldots, x_{k} \in$ $\widetilde{H}_{*}(X), \lambda_{1}, \ldots, \lambda_{t}$ are Browder products of weight $l_{1}, \ldots, l_{t}$ respectively, and $2 \leq k+l_{1}+\cdots+l_{t} \leq p$. Since $\eta_{*}$ and $\left(\eta_{p}\right)_{*}$ are one-to-one, we can by abuse of notation consider $B \oplus C$ as a decomposition of $\widetilde{H}_{*}\left(\widetilde{C}_{n, p} X\right)$. We can then make

Definition 3.2. If $v \in \widetilde{H}_{q}(X)$ and $q+s$ is even then the external homology Dyer-Lashof operation $\bar{Q}_{s(p-1)-\gamma} v \in \widetilde{H}_{*}\left(\widetilde{C}_{n, p} X\right) \subset \widetilde{H}_{*}\left(C_{n, p} X\right)$ is defined as $Q_{s(p-1)-\gamma} \eta_{*}(v)$. 
If $\left(X, \theta_{n}^{X}\right) \in \mathrm{Ob} \mathcal{H}_{p}^{n}$, then the structure map $\theta_{n}^{X}$ characterizes the internal Dyer-Lashof operations in $\widetilde{H}_{*}(X)$ by the formula

$$
Q_{r} v=\left(\theta_{n}^{X}\right)_{*}\left(\bar{Q}_{r} v\right) \text {. }
$$

We also have

$$
h_{*}\left(\sigma^{n} \bar{Q}_{r} v\right)=\sigma^{n} Q_{r} v .
$$

To see this, first note that $\left(\varepsilon_{n}\right)_{*}\left(\bar{Q}_{r} v\right)=0$ for otherwise the formula $\left(\varepsilon_{n}\right)_{*}\left(\bar{Q}_{r} v\right)$ would define a natural positive-dimensional homology operation on an arbitrary space, which is impossible. The formula then follows by a simple diagram chase on the diagram from Section 2 defining $h$.

Now we come to the maps $G$ and $\tilde{\varepsilon}_{k}$. From the point of view of the theory of spectra, one way to think about $G$ is that, when $\gamma=0$, the existence of the $G$ we want is essentially equivalent to the fact that the $\bmod p$ Eilenberg-MacLane spectrum admits the structure of an $H_{\infty}^{2}$ ring spectrum (as defined by Bruner et. al. [BMMS]). In [KSW], the analogous key fact is the existence of an $H_{\infty}^{1}$ ring spectrum structure on the mod 2 Eilenberg-MacLane spectrum. In fact, using the methods of this paper and of $[\mathbf{K S W}]$ it should be possible to generalize our main theorem to a theorem on arbitrary $H_{\infty}^{d}$ ring spectrums $E$, which would relate power operations in the $E^{*}$ cohomology of a generalized projective plane to power operations in the $E_{*}$ homology of the original space.

For our purposes, it is convenient to have an explicit description of $G$. The main technical difficulty in the odd primary case which does not cause a problem in the 2 primary case in $[\mathbf{K S W}]$ is the fact that $\widetilde{C}_{\infty, p} X$ is not homotopy equivalent to the p-adic construction, $D_{p} X$, when $p$ is odd (stably it contains $D_{p} X$ as a wedge summand), whereas it is when $p=2$. This leads to many possible choices for the definition of $G$ in the odd primary case, and it is important to carefully define the "correct" one.

To simplify notation let $K=K(\mathbf{Z} / p, s+q-\gamma)$. Then the fundamental class $\iota=\iota_{s+q-\gamma}$, its images under the Steenrod algebra, and their respective products form a standard basis $\mathcal{B} \widetilde{H}^{*}(K)$ for $\widetilde{H}^{*}(K)$; let its dual basis be $\mathcal{B} \widetilde{H}_{*}(K)$, and let the element of $\mathcal{B} \widetilde{H}_{*}(K)$ dual to $\iota$ be $\nu$.

By Theorem $3.1, \widetilde{H}_{*}\left(\widetilde{C}_{\infty, p} K\right) \cong F_{p} \widetilde{H}_{*}\left(C_{\infty} K\right) / F_{1} \widetilde{H}_{*}\left(C_{\infty} K\right)$. If we then apply the structure theorem for $\widetilde{H}_{*}\left(C_{\infty} X\right)$ ([CLM, I, 4.1]), it is not hard to show that one gets a basis for $\widetilde{H}_{*}\left(\widetilde{C}_{\infty, p} K\right)$ by totally ordering the elements of $\mathcal{B} \widetilde{H}_{*}(K)$ and then defining the set $\mathcal{B} \widetilde{H}_{*}\left(\widetilde{C}_{\infty, p} K\right)$ to be $A \cup B$, where

$$
\begin{array}{r}
A=\left\{\eta_{*}\left(v_{1}\right) * \cdots * \eta_{*}\left(v_{k}\right) \mid v_{1}, \ldots,\right. \\
\left.\quad v_{k} \in \mathcal{B} \widetilde{H}_{*}(K), 2 \leq k \leq p, v_{1} \leq v_{2} \leq \cdots \leq v_{k}\right\}, \\
B=\left\{\bar{Q}_{s(p-1)-\gamma} v \mid v \in \mathcal{B} \widetilde{H}_{*}(K),(s+\operatorname{deg} v) \text { even, } s>0, \gamma=0 \text { or } 1\right\} .
\end{array}
$$


We emphasize that the notation $\eta_{*}\left(v_{1}\right) * \cdots * \eta_{*}\left(v_{k}\right)$ for an element of $\widetilde{H}_{*}\left(\widetilde{C}_{\infty, p} K\right)$ only makes sense via the isomorphism with

$$
F_{p} \widetilde{H}_{*}\left(C_{\infty} K\right) / F_{1} \widetilde{H}_{*}\left(C_{\infty} K\right)
$$

since $\widetilde{C}_{\infty, p} K$ itself is not an H-space and thus admits no Pontryagin products.

We now let $\mathcal{B} \widetilde{H}^{*}\left(\widetilde{C}_{\infty, p} K\right)$ be the dual basis to $\mathcal{B} \widetilde{H}_{*}\left(\widetilde{C}_{\infty, p} K\right)$, and, for each $v$ in $\mathcal{B} \widetilde{H}_{*}\left(\widetilde{C}_{\infty, p} K\right)$, we denote the dual basis element by $(v)^{\text {dual }}$. If $\gamma=0$ we then define $G: \widetilde{C}_{\infty, p} K(\mathbf{Z} / p, s+q) \rightarrow K(\mathbf{Z} / p, p(s+q))$ to be the map representing the cohomology class

$$
\sum\left(\eta_{*}\left(\nu^{t_{1}}\right) * \cdots * \eta_{*}\left(\nu^{t_{k}}\right)\right)^{\text {dual }}
$$

where the sum runs over sequences $\left(t_{1}, \ldots, t_{k}\right)$ such that

1. $k \leq p$,

2. $t_{1}+\cdots+t_{k}=p$, and

3. $\nu^{t_{1}} \leq \nu^{t_{2}} \leq \cdots \leq \nu^{t_{k}}$ in the ordering on $\mathcal{B} \widetilde{H}_{*}(K)$.

This definition makes sense since we have required that $s+q$ be even.

If $\gamma=1$ then we define $G: \widetilde{C}_{\infty, p} K(\mathbf{Z} / p, s+q-1) \rightarrow K(\mathbf{Z} / p, p(s+q)-2)$ to be the map representing the cohomology class

$$
\left(\bar{Q}_{p-2} \nu\right)^{\text {dual }} \text {. }
$$

Now we consider the map $\tilde{\varepsilon}_{k}$. It is constructed in [KSW], and it fits into a commutative diagram

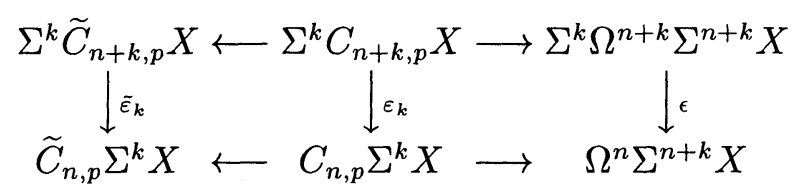

where $\varepsilon_{k}$ is a map constructed in [Ma2] and $\epsilon: \Sigma^{k} \Omega^{k} Y \rightarrow Y$ is the evaluation map. If $X$ is connected, so that $C_{n} X \simeq \Omega^{n} \Sigma^{n} X$, then $\varepsilon_{k}$ may be seen as "filtering" $\epsilon$.

One important fact about $\tilde{\varepsilon}_{k}$ is that it commutes with the (homology) Dyer-Lashof operations in the sense that

$$
\left(\tilde{\varepsilon}_{k}\right)_{*}\left(\sigma^{k} \bar{Q}_{s(p-1)-\gamma} v\right)= \begin{cases}\bar{Q}_{(s-k)(p-1)-\gamma} \sigma^{k} v & \text { if } s-k>\gamma \\ 0 & \text { otherwise }\end{cases}
$$

This follows from the above commutative diagram combined with the standard fact that the Dyer-Lashof operations commute with the loop homomorphism induced by $\epsilon$ (see, e.g., [CLM, III, Theorem 1.4]). We also note that $\tilde{\varepsilon}_{k}$ is still defined when $n=\infty$. 
We now define the external cohomology operations.

Definition 3.3. Suppose $\gamma$ is an element of the set $\{0,1\}$, and $s \geq \gamma$ and $q \geq 0$ are integers such that $s+q$ is even. Let $r=s(p-1)-\gamma$. For every $1 \leq n \leq \infty$ and any space $X$, let

$$
\widetilde{Q}_{r}: \widetilde{H}^{q}(X) \longrightarrow \widetilde{H}^{p q+r}\left(\widetilde{C}_{n, p} X\right)
$$

be defined as follows. If $x \in \widetilde{H}^{q}(X)$ is represented by $f: X \rightarrow K(\mathbf{Z} / p, q)$, let $\sigma^{s-\gamma}\left(\widetilde{Q}_{r} x\right)$ be represented by the composite

$$
\begin{aligned}
& \quad \Sigma^{s-\gamma} \widetilde{C}_{n, p} X \rightarrow \Sigma^{s-\gamma} \widetilde{C}_{\infty, p} X \stackrel{\tilde{\varepsilon}_{s-\gamma}}{\longrightarrow} \widetilde{C}_{\infty, p} \Sigma^{s-\gamma} X \stackrel{\widetilde{C}_{\infty, p} \Sigma^{s-\gamma} f}{\longrightarrow} \widetilde{C}_{\infty, p} \Sigma^{s-\gamma} K(\mathbf{Z} / p, q) \\
& \quad \stackrel{\widetilde{C}_{\infty, p} \epsilon}{\longrightarrow} \widetilde{C}_{\infty, p} K(\mathbf{Z} / p, s+q-\gamma) \stackrel{G}{\longrightarrow} K(\mathbf{Z} / p, p(s+q)-2 \gamma) .
\end{aligned}
$$

The element $\widetilde{Q}_{r} x$ for $r>0$ is sometimes denoted $e_{r} \otimes x^{p}$ or $e_{r} \int x$ in the literature (the way our operations are defined, $\widetilde{Q}_{0} x \neq e_{0} \int x$ ). We remark for future reference that when $s \geq n$ above, the operation $\widetilde{Q}_{r}$ is trivial.

The next proposition relates our external cohomology operations $\widetilde{Q}_{r}$ to the external homology operations, via the Kronecker pairing $\langle$,$\rangle . This$ will make it reasonable to see $\widetilde{Q}_{r}$ as a sort of "dual external Dyer-Lashof operation".

Proposition 3.4. Suppose $x \in \widetilde{H}^{q}(X)$ and $v \in \widetilde{H}_{q}(X)$. Then

$$
\left\langle\widetilde{Q}_{r} x, \bar{Q}_{r^{\prime}} v\right\rangle= \begin{cases}\langle x, v\rangle & \text { if } r=r^{\prime} \\ 0 & \text { if } r \neq r^{\prime}\end{cases}
$$

Furthermore, if $r=s(p-1)-\gamma>0$, then the element $\widetilde{Q}_{r} x$ paired with any Browder or Pontryagin product operation yields zero.

Proof. If $r>0$ we assume by naturality that $n=\infty$ and make the following calculation. By the definition of $\widetilde{Q}_{r} x$,

$$
\begin{aligned}
\left\langle\widetilde{Q}_{r} x, \bar{Q}_{r^{\prime}} v\right\rangle & =\left\langle\sigma^{s-\gamma} \widetilde{Q}_{s(p-1)-\gamma} x, \sigma^{s-\gamma} \bar{Q}_{s^{\prime}(p-1)-\gamma^{\prime}} v\right\rangle \\
& =\left\langle\tilde{\varepsilon}_{s-\gamma}^{*}\left(\widetilde{C}_{\infty, p} \Sigma^{s-\gamma} f\right)^{*}\left(\widetilde{C}_{\infty, p} \epsilon\right)^{*}[G], \sigma^{s-\gamma} \bar{Q}_{s^{\prime}(p-1)-\gamma^{\prime}} v\right\rangle \\
& =\left\langle[G],\left(\widetilde{C}_{\infty, p} \epsilon\right)_{*}\left(\widetilde{C}_{\infty, p} \Sigma^{s-\gamma} f\right)_{*}\left(\tilde{\varepsilon}_{s-\gamma}\right)_{*} \sigma^{s-\gamma} \bar{Q}_{s^{\prime}(p-1)-\gamma^{\prime}} v\right\rangle .
\end{aligned}
$$

Now, we have already observed how $\left(\tilde{\varepsilon}_{s-\gamma}\right)_{*}$ commutes with the $\bar{Q}_{r}$. In addition, for any map $g: X \rightarrow Y$, we have $\left(C_{\infty} g\right)_{*}\left(Q_{r} \eta_{*} v\right)=Q_{r} g_{*}\left(\eta_{*} v\right)$ [CLM], and hence

$$
\left(\widetilde{C}_{\infty, p} g\right)_{*}\left(\bar{Q}_{r} v\right)=\bar{Q}_{r} g_{*}(v)
$$


Thus,

$$
\begin{aligned}
\left\langle[G],\left(\widetilde{C}_{\infty, p} \epsilon\right)_{*}\left(\widetilde{C}_{\infty, p} \Sigma^{s-\gamma} f\right)_{*}\left(\tilde{\varepsilon}_{s-\gamma}\right)_{*} \sigma^{s-\gamma} \bar{Q}_{s^{\prime}(p-1)-\gamma^{\prime}} v\right\rangle \\
=\left\langle[G], \bar{Q}_{\left(s^{\prime}-s+\gamma\right)(p-1)-\gamma^{\prime}} \epsilon_{*} \sigma^{s-\gamma} f_{*}(v)\right\rangle .
\end{aligned}
$$

Here we let $\bar{Q}_{r} x=0$ if $r<0$. Now, by the construction of $G$, the last quantity can be nonzero only if $\bar{Q}_{\left(s^{\prime}-s+\gamma\right)(p-1)-\gamma^{\prime}} \epsilon_{*} \sigma^{s-\gamma} f_{*}(v)$ equals either $u \cdot \bar{Q}_{0} \nu_{s+q}$ or $u \cdot \bar{Q}_{p-2} \nu_{s+q-1}$, where $u \in \mathbf{F}_{p}$ is some unit. In the former case, $\gamma=\gamma^{\prime}=0$ and $s-s^{\prime}=0$, and in the latter case, $\operatorname{deg}\left(\epsilon_{*} \sigma^{s-\gamma} f_{*}(v)\right)=s+q-\gamma$ must be odd for the Dyer-Lashof operation to be nontrivial, implying (since $s+q$ is even) that $\gamma=1$. Thus $\gamma=\gamma^{\prime}=1$ and $s-s^{\prime}=0$, and so in either case $s^{\prime}=s$ and $\gamma^{\prime}=\gamma$, i.e., $r^{\prime}=r$.

Now let us write $r^{\prime \prime}$ for $\left(s^{\prime}-s+\gamma\right)(p-1)-\gamma^{\prime}=\gamma(p-1)-\gamma$. Then $\left\langle[G], u \cdot \bar{Q}_{r^{\prime \prime}} \nu_{s+q-\gamma}\right\rangle=u$, so we need to show that

$$
\bar{Q}_{r^{\prime \prime}} \epsilon_{*} \sigma^{s-\gamma} f_{*}(v)=\langle x, v\rangle \cdot \bar{Q}_{r^{\prime \prime}} \nu_{s+q-\gamma} .
$$

By linearity of the Dyer-Lashof operations it suffices to show that $\epsilon_{*} \sigma^{s-\gamma} f_{*}(v)=\langle x, v\rangle \cdot \nu_{s+q-\gamma}$, and, since $H_{s+q-\gamma}(K(\mathbf{Z} / p, s+q-\gamma))$ is one-dimensional, this amounts to showing that $\left\langle\iota_{s+q-\gamma}, \epsilon_{*} \sigma^{s-\gamma} f_{*}(v)\right\rangle=\langle x, v\rangle$. This is easily seen to be true.

For the other formulas we are assuming $r>0$, which means that $\widetilde{Q}_{r} x$ is in the kernel of the map $\widetilde{H}^{*}\left(\widetilde{C}_{n, p} X\right) \rightarrow \widetilde{H}^{*}\left(\widetilde{C}_{1, p} X\right)$ induced by inclusion. Thus the pairing of $\widetilde{Q}_{r} x$ with any Pontryagin product operation applied to $(v, w)$ must be zero. Similarly, since $\widetilde{Q}_{r} x$ comes from the cohomology of $\widetilde{C}_{\infty, p} X$, it must pair trivially with any Browder operation.

It follows from Proposition 3.4, along with the formula for $h_{*}\left(\sigma^{n} \bar{Q}_{r} v\right)$, that if $h^{*}\left(\sigma^{n} y\right)=\sigma^{n} \widetilde{Q}_{r} x$ for some $x, y \in \widetilde{H}^{*}(X)$, then $\langle x, v\rangle=\left\langle y, Q_{r} v\right\rangle$ for all $v \in \widetilde{H}_{*}(X)$. Equivalently, if $h^{*}\left(\sigma^{n} y\right)=\sigma^{n} \widetilde{Q}_{r} x$, then $Q_{r}^{*} y=x$ as in Part 1 of the Main Theorem.

We close this section with the following lemma, which shows that the $\widetilde{Q}_{r}$ commute with $\tilde{\varepsilon}_{k}^{*}$ just as one would want.

Lemma 3.5. Let $k>0$ be an integer, let $X$ be an $H_{p}^{n+k}$-space, and choose $x \in \widetilde{H}^{q}(X)$. Then

$$
\tilde{\varepsilon}_{k}^{*}\left(\widetilde{Q}_{s(p-1)-\gamma} \sigma^{k} x\right)=\sigma^{k} \widetilde{Q}_{(s+k)(p-1)-\gamma} x
$$

for any $\gamma \leq s<n+k$. 
Proof. As with Proposition 3.4, we assume $n=\infty$ by naturality, and we will show that

$$
\sigma^{q-\gamma} \tilde{\varepsilon}_{k}^{*}\left(\widetilde{Q}_{s(p-1)-\gamma} \sigma^{k} x\right)=\sigma^{q-\gamma+k} \widetilde{Q}_{(s+k)(p-1)-\gamma} x .
$$

Let $x$ be represented by the map $f: X \rightarrow K(\mathbf{Z} / p, q)$, in which case $\sigma^{k} x$ will be represented by the map $\epsilon \circ \Sigma^{k} f: \Sigma^{k} X \rightarrow K(\mathbf{Z} / p, q+k)$. Then, by definition

$$
\sigma^{q-\gamma} \tilde{\varepsilon}_{k}^{*}\left(\widetilde{Q}_{s(p-1)-\gamma} \sigma^{k} x\right)=\left(\Sigma^{s-\gamma} \tilde{\varepsilon}_{k}\right)^{*} \tilde{\varepsilon}_{s-\gamma}^{*}\left(\widetilde{C}_{\infty, p} \Sigma^{s-\gamma}\left(\epsilon \circ \Sigma^{k} f\right)\right)^{*}\left(\widetilde{C}_{\infty, p} \epsilon\right)^{*}[G] .
$$

Now, $\tilde{\varepsilon}_{j} \circ \Sigma^{j} \tilde{\varepsilon}_{k}=\tilde{\varepsilon}_{j+k}$ by construction (see $[\mathbf{K S W}]$ ), and

$$
\widetilde{C}_{\infty, p} \Sigma^{s-\gamma}\left(\epsilon \circ \Sigma^{k} f\right)=\widetilde{C}_{\infty, p} \Sigma^{s-\gamma} \epsilon \circ \widetilde{C}_{\infty, p} \Sigma^{k+s-\gamma} f
$$

by functoriality, so we actually have

$$
\sigma^{q-\gamma} \tilde{\varepsilon}_{k}^{*}\left(\widetilde{Q}_{s(p-1)-\gamma} \sigma^{k} x\right)=\tilde{\varepsilon}_{k+s-\gamma}^{*}\left(\widetilde{C}_{\infty, p} \Sigma^{k+s-\gamma} f\right)^{*}\left(\widetilde{C}_{\infty, p} \Sigma^{s-\gamma} \epsilon\right)^{*}\left(\widetilde{C}_{\infty, p} \epsilon\right)^{*}[G] .
$$

But then $\epsilon \circ \Sigma^{s-\gamma} \epsilon=\epsilon$, so in fact

$$
\begin{aligned}
\sigma^{q-\gamma} \tilde{\varepsilon}_{k}^{*}\left(\widetilde{Q}_{s(p-1)-\gamma} \sigma^{k} x\right) & =\tilde{\varepsilon}_{k+s-\gamma}^{*}\left(\widetilde{C}_{\infty, p} \Sigma^{k+s-\gamma} f\right)^{*}\left(\widetilde{C}_{\infty, p} \epsilon\right)^{*}[G] \\
& =\sigma^{q-\gamma+k} \widetilde{Q}_{(s+k)(p-1)-\gamma} x
\end{aligned}
$$

\section{The Main Theorem.}

Part 1 of the Main Theorem has already been proved; here we restate Part 2 in slightly altered form. First we introduce the following convention. Suppose $x_{1}$ and $x_{2}$ are two elements of an $\mathbf{F}_{p}$-algebra (e.g. the mod $p$ cohomology of a space). Then we say $x_{1} \doteq x_{2}$ if $x_{1}=u \cdot x_{2}$ where $u \in \mathbf{F}_{p}$ is a unit. This is easily seen to be an equivalence relation.

Theorem 4.1. Suppose $p$ is an odd prime and $X \in \mathrm{ObH}_{p}^{n}$, with basic cofibration sequence

$$
\Sigma^{n} \widetilde{C}_{n, p} X \stackrel{h}{\longrightarrow} \Sigma^{n} X \stackrel{\imath}{\longrightarrow} P_{p}^{n} X \stackrel{j}{\longrightarrow} \Sigma^{n+1} \widetilde{C}_{n, p} X .
$$

Let $n=2 s+1-\delta$ for some $\delta \in\{0,1\}$ and $s \geq \delta$. If $\bar{x} \in \widetilde{H}^{2 q+\delta+n}\left(P_{p}^{n} X\right)$, then

$$
\begin{aligned}
\mathcal{P}^{q+s} \bar{x} & \doteq j^{*}\left(\sigma^{n+1} \widetilde{Q}_{(n-1)(p-1)-1} x\right), \\
\beta \mathcal{P}^{q+s} \bar{x} & \doteq j^{*}\left(\sigma^{n+1} \widetilde{Q}_{(n-1)(p-1)} x\right),
\end{aligned}
$$


where $i^{*} \bar{x}=\sigma^{n} x$ defines $x \in \widetilde{H}^{2 q+\delta}(X)$.

We first prove Theorem 4.1 for the universal example $X=K(\mathbf{Z} / p, 2 q+\delta)$ and $x=\iota_{2 q+\delta}$; the universal example is then used to prove the theorem for general $X$. We will prove the formulas for $\beta \mathcal{P}^{q+s} \bar{\iota}_{2 q+\delta}$ and $\mathcal{P}^{q+s} \bar{\iota}_{2 q+\delta}$ separately.

For the $\beta \mathcal{P}^{q+s} \bar{\iota}_{2 q+\delta}$ formula we first consider the special case in which $s=$ $\delta=0$. Let $\bar{\iota}_{2 q} \in \widetilde{H}^{2 q+1}\left(P_{p}^{1} K(\mathbf{Z} / p, 2 q+\delta)\right)$ be (the unique class) such that $i^{*}\left(\bar{\iota}_{2 q}\right)=\iota_{2 q}$; we know such a class exists because $\iota_{2 q}$ is representable by an infinite loop map, and hence $h^{*}\left(\iota_{2 q}\right)=0$. We use the following commutative diagram, where $K(\mathbf{Z} / p, t)$ is written $K_{t}, \kappa$ represents $\beta \mathcal{P}^{q} \iota_{2 q+1}$, and $E$ is the homotopy fiber of $\kappa$.

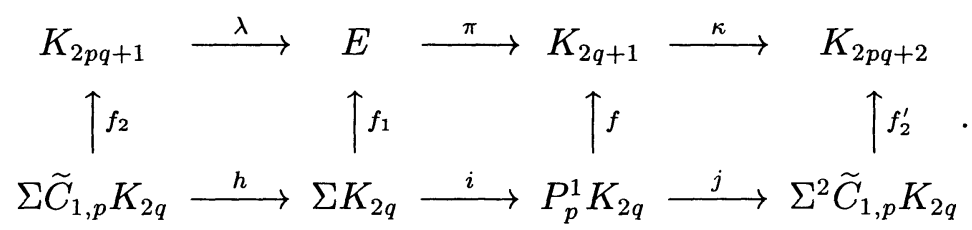

Here the map $f$ represents the class $\bar{\iota}_{2 q}$, and the map $f_{1}$ exists by the lifting property of a fibration since $\kappa f i$ represents

$$
\beta \mathcal{P}^{q} i^{*}\left(\bar{\iota}_{2 q}\right)=\beta \mathcal{P}^{q} \iota_{2 q}=0
$$

and is thus homotopically trivial. There is of course some indeterminacy in the choice of $f_{1}$; we will begin with an arbitrary choice and then alter it as necessary by maps factoring through the fiber.

We will give a specific construction of $f_{2}$ making the leftmost square commute, and then we can choose $f_{2}^{\prime}$ to be the adjoint of $f_{2}$. This will make the rightmost square commute as well. (We will in general let $g^{\prime}$ denote the adjoint of $g$, where $g$ may either be a map $X \rightarrow \Omega Y$ or $\Sigma X \rightarrow Y$.) The construction of $f_{2}$ will in turn be as the adjoint of a map $\widetilde{C}_{1, p} K_{2 q} \rightarrow K_{2 p q}$ factoring through $\Omega E$, and the key to the proof is that the image of $\iota_{2 p q}$ in $H^{*}(\Omega E)$ is connected by the opposite Dyer-Lashof operation $Q_{0}^{*}$ to $(\Omega \pi)^{*}\left(\iota_{2 q}\right)$, and that this connection is preserved by the map $\widetilde{C}_{1, p} K_{2 q} \rightarrow \Omega E$.

First let us construct $f_{2}$. Because $\Omega \kappa$ is homotopically trivial, $\Omega E \simeq$ $K_{2 p q} \times K_{2 q}$. Hence there is a map $\rho: \Omega E \rightarrow K_{2 p q}$ such that $\rho \Omega \lambda$ is homotopic to the identity. We then define $f_{2}$ to be the adjoint of the composition

$$
\widetilde{C}_{1, p} K_{2 q} \stackrel{h^{\prime}}{\longrightarrow} \Omega \Sigma K_{2 q} \stackrel{\Omega f_{1}}{\longrightarrow} \Omega E \stackrel{\rho}{\longrightarrow} K_{2 p q} .
$$

Thus, if we write $\rho^{*}\left(\iota_{2 p q}\right)$ as just $\iota_{2 p q} \in \widetilde{H}^{*}(\Omega E)$, then $f_{2}$ represents $\sigma\left(h^{\prime}\right)^{*}\left(\Omega f_{1}\right)^{*} \iota_{2 p q}$. Using the definitions of the various maps and the relationships between them, we then see that

$$
\beta \mathcal{P}^{q} \bar{\iota}=j^{*}\left(\sigma^{2}\left(h^{\prime}\right)^{*}\left(\Omega f_{1}\right)^{*} \iota_{2 p q}\right) .
$$


Hence to prove the theorem in this case we need only show that

$$
\left(h^{\prime}\right)^{*}\left(\Omega f_{1}\right)^{*} \iota_{2 p q} \doteq \widetilde{Q}_{0} \iota_{2 q} .
$$

We will do this by showing that

$$
\left\langle\left(h^{\prime}\right)^{*}\left(\Omega f_{1}\right)^{*} \iota_{2 p q}, v\right\rangle \doteq\left\{\begin{array}{cc}
1 & \text { if } v=\eta_{*}\left(\nu_{2 q}^{t_{1}}\right) * \cdots * \eta_{*}\left(\nu_{2 q}^{t_{k}}\right) \\
& \text { with } t_{1}+\cdots+t_{k}=p \\
0 & \text { otherwise }
\end{array}\right.
$$

for any $v$ in the basis

$$
\begin{aligned}
\mathcal{B} \widetilde{H}_{*}\left(\widetilde{C}_{1, p} K_{2 q}\right) & \\
& =\left\{\eta_{*}\left(v_{1}\right) * \cdots * \eta_{*}\left(v_{k}\right) \mid v_{1}, \ldots, v_{k} \in \mathcal{B} \widetilde{H}_{*}\left(K_{2 q}\right), 2 \leq k \leq p\right\} .
\end{aligned}
$$

It is easy to see that this characterizes $\widetilde{Q}_{0} \iota_{2 q}$.

We will be using the fact that $\left\langle\left(h^{\prime}\right)^{*}\left(\Omega f_{1}\right)^{*} \iota_{2 p q}, v\right\rangle=\left\langle\iota_{2 p q},\left(\Omega f_{1}\right)_{*}\left(h^{\prime}\right)_{*} v\right\rangle$, and so we begin by considering $\iota_{2 p q}$. We claimed above that $\iota_{2 p q}$ was connected to $\iota_{2 q}$ by $Q_{0}^{*}$. More precisely, let $\mathcal{B} \widetilde{H}^{*}(\Omega E)$ be the obvious basis consisting of products of elements of $\mathcal{B} \widetilde{H}^{*}(K(\mathbf{Z} / p, 2 q))$ with elements of $\mathcal{B} \widetilde{H}^{*}(K(\mathbf{Z} / p, 2 p q))$, and let $\mathcal{B} \widetilde{H}_{*}(\Omega E)$ be the dual basis. Then

$$
\left(\nu_{2 q}^{p}\right)^{\text {dual }} \doteq \iota_{2 p q} .
$$

This follows from the well-known formula for the coproduct of $\iota_{2 p q}$ in $H^{*}(E)$; see [Za, §3]. We cannot simply choose a representation of $\Omega E$ as $K_{2 q} \times K_{2 p q}$ to make the equality exact as does Zabrodsky, since the projection $\rho: \Omega E \rightarrow$ $K_{2 p q}$ is constrained by the requirement that it be a retraction of the map $\Omega \lambda$.

Since $\left(\nu_{2 q}^{p}\right)^{\text {dual }} \doteq \iota_{2 p q}$, it will suffice to show, for $v \in \mathcal{B} \widetilde{H}_{*}\left(\widetilde{C}_{1, p} K_{2 q}\right)$, that $\left(\Omega f_{1}\right)_{*}\left(h^{\prime}\right)_{*} v \doteq \nu_{2 q}^{p}$ if and only if $v \doteq \eta_{*}\left(\nu_{2 q}^{t_{1}}\right) * \cdots * \eta_{*}\left(\nu_{2 q}^{t_{k}}\right)$ with $t_{1}+\cdots+t_{k}=p$. We will prove the following formula:

$$
\left(\Omega f_{1}\right)_{*}\left(h^{\prime}\right)_{*}\left(\eta_{*}\left(v_{1}\right) * \cdots * \eta_{*}\left(v_{k}\right)\right)=-v_{1} * \cdots * v_{k},
$$

which does the trick.

We begin with $\left(h^{\prime}\right)_{*}$. By considering how $h$ was constructed (Section 2, see [KSW] for details), we see that its adjoint $h^{\prime}$ fits into the following commutative diagram.

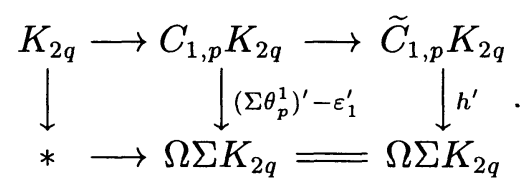


Here we note that $\varepsilon_{1}^{\prime}$ is the map

$$
C_{1, p} K_{2 q} \hookrightarrow C_{1} K_{2 q} \stackrel{\simeq}{\longrightarrow} \Omega \Sigma K_{2 q},
$$

where the second arrow is the usual equivalence of May [Ma2].

Let $\eta_{*}\left(v_{1}\right) * \cdots * \eta_{*}\left(v_{k}\right) \in \mathcal{B} \widetilde{H}_{*}\left(\widetilde{C}_{1, p} K_{2 q}\right)$ be given, and observe, since $\left(\varepsilon_{1}^{\prime}\right)_{*}$ is operation preserving, that

$\left(h^{\prime}\right)_{*}\left(\eta_{*}\left(v_{1}\right) * \cdots * \eta_{*}\left(v_{k}\right)\right)=\left(\Sigma \theta_{p}^{1}\right)_{*}^{\prime}\left(\eta_{*}\left(v_{1}\right) * \cdots * \eta_{*}\left(v_{k}\right)\right)-\eta_{*}\left(v_{1}\right) * \cdots * \eta_{*}\left(v_{k}\right)$.

Now $\left(\Sigma \theta_{p}^{1}\right)^{\prime}$ is the composition

$$
C_{1, p} K_{2 q} \stackrel{\eta}{\longrightarrow} \Omega \Sigma C_{1, p} K_{2 q} \stackrel{\Omega \Sigma \theta_{p}^{1}}{\longrightarrow} \Omega \Sigma K_{2 q},
$$

implying that $\left(\Sigma \theta_{p}^{1}\right)_{*}^{\prime}\left(\eta_{*}\left(v_{1}\right) * \cdots * \eta_{*}\left(v_{k}\right)\right)=\eta_{*}\left(v_{1} * \cdots * v_{k}\right)$ and hence

$$
\left(h^{\prime}\right)_{*}\left(\eta_{*}\left(v_{1}\right) * \cdots * \eta_{*}\left(v_{k}\right)\right)=\eta_{*}\left(v_{1} * \cdots * v_{k}\right)-\eta_{*}\left(v_{1}\right) * \cdots * \eta_{*}\left(v_{k}\right) .
$$

We will handle the two terms on the right hand separately, first altering $f_{1}$ by a map factoring through $K_{2 p q}$ so that $\left\langle\iota_{2 p q},\left(\Omega f_{1}\right)_{*} \eta_{*}\left(v_{1} * \cdots * v_{k}\right)\right\rangle=0$. For each $v \in \mathcal{B} \widetilde{H}_{2 p q}\left(K_{2 q}\right)$, let $f_{v}: \Sigma K_{2 q} \rightarrow K_{2 p q+1}$ be the map representing the cohomology class $\left\langle\left(\Omega f_{1}\right)^{*} \iota_{2 p q}, \eta_{*}(v)\right\rangle \cdot \sigma\left(v^{\text {dual }}\right)$, and take $f_{1}$ now to be the map obtained from the original choice of $f_{1}$ by subtracting the sum

$$
\sum_{v \in \mathcal{B} H_{2 p q}\left(K_{2 q}\right)} \lambda \circ f_{v}
$$

using the $\mathrm{H}$-structure of $E$. Redefine $f_{2}$ accordingly. Then

$$
\left\langle\iota_{2 p q},\left(\Omega f_{1}\right)_{*} \eta_{*}(v)\right\rangle=0
$$

for any $v$ in $\mathcal{B} \widetilde{H}_{2 p q}\left(K_{2 q}\right)$ by the construction of $f_{1}$, and in particular,

$$
\left\langle\iota_{2 p q},\left(\Omega f_{1}\right)_{*} \eta_{*}\left(v_{1} * \cdots * v_{k}\right)\right\rangle=0 .
$$

We now have to consider $\left\langle\iota_{2 p q},\left(\Omega f_{1}\right)_{*}\left(-\eta_{*}\left(v_{1}\right) * \cdots * \eta_{*}\left(v_{k}\right)\right)\right\rangle$. By construction,

$$
(\Omega \pi)\left(\Omega f_{1}\right) \eta \simeq(\Omega f)(\Omega i) \eta \simeq \mathrm{id},
$$

and hence $\left(\Omega f_{1}\right)_{*} \eta_{*}(v)=v$ for any $v \in H_{*}\left(K_{2 q}\right)$, where, on the right hand side, $v$ denotes $1 \otimes v$ in $H_{*}(\Omega E)$. Since $\Omega f_{1}$ is an H-map, we see thàt $\left(\Omega f_{1}\right)_{*}\left(-\eta_{*}\left(v_{1}\right) * \cdots * \eta_{*}\left(v_{k}\right)\right)=-v_{1} * \cdots * v_{k} \in \widetilde{H}^{2 p q}(\Omega E)$. Thus, putting together our calculations of $\left(\Omega f_{1}\right)_{*}$ and $\left(h^{\prime}\right)_{*}$, we have $\left(\Omega f_{1}\right)_{*}\left(h^{\prime}\right)_{*}\left(\eta_{*}\left(v_{1}\right) *\right.$ $\left.\cdots * \eta_{*}\left(v_{k}\right)\right)=-v_{1} * \cdots * v_{k}$ for any $\eta_{*}\left(v_{1}\right) * \cdots * \eta_{*}\left(v_{k}\right) \in \mathcal{B} \widetilde{H}_{*}\left(\widetilde{C}_{1, p} K_{2 q}\right)$, as we wanted. 
Consider now the case in which $\delta=0$ or 1 and $s>1$. Theorem 4.1 of $[\mathbf{K S W}]$ implies that the following diagram commutes up to homotopy.

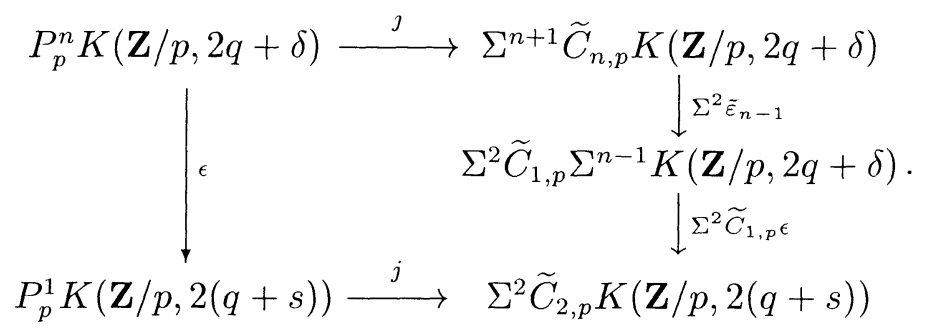

Here, $\epsilon$ represents one of two natural transformations, running either

$$
P_{p}^{n+k} \Omega^{n} \rightarrow P_{p}^{k} \quad \text { or } \quad \Sigma^{n+k} \Omega^{n} \rightarrow \Sigma^{k},
$$

which are determined by the adjointness of $P_{p}^{n}$ and $\Sigma^{n}$ with $\Omega^{n}$, respectively. Since the choices of $\bar{\iota}_{2 q+1}$ and $\bar{\iota}_{2(q+s-1)+1}$ are unique, one sees that $\epsilon^{*}\left(\bar{\iota}_{2(q+s)}\right)=\bar{\iota}_{2 q+\delta}$, and hence $\epsilon^{*}\left(\beta \mathcal{P}^{q+s} \bar{\iota}_{2(q+s)}\right)=\beta \mathcal{P}^{q+s} \bar{\iota}_{2 q+\delta}$. By Lemma 3.5,

$$
\left(\Sigma^{2}\left(\widetilde{C}_{1, p} \epsilon \circ \tilde{\varepsilon}_{n-1}\right)\right)^{*}\left(\sigma^{2} \widetilde{Q}_{0} \iota_{2(q+s)}\right)=\sigma^{n+1} \widetilde{Q}_{(n-1)(p-1)} \iota_{2 q+\delta} ;
$$

thus

$$
\begin{aligned}
j^{*}\left(\sigma^{n+1} \widetilde{Q}_{(n-1)(p-1)} \iota_{2 q+\delta}\right) & =j^{*}\left(\Sigma^{2}\left(\widetilde{C}_{1, p} \epsilon \circ \tilde{\varepsilon}_{n-1}\right)\right)^{*}\left(\sigma^{2} \widetilde{Q}_{0} \iota_{2(q+s)}\right) \\
& =\epsilon^{*} j^{*}\left(\sigma^{2} \widetilde{Q}_{0} \iota_{2(q+s)}\right) \\
& \doteq \epsilon^{*}\left(\beta \mathcal{P}^{q+s} \bar{\iota}_{2(q+s)}\right) \\
& =\beta \mathcal{P}^{q+s} \bar{\iota}_{2 q+\delta} .
\end{aligned}
$$

In order to prove the formula $\mathcal{P}^{q+s} \bar{\iota}_{2 q+\delta} \doteq j^{*}\left(\sigma^{n+1} \widetilde{Q}_{(n-1)(p-1)-1} \iota_{2 q+\delta}\right)$ for $s>0$, we need only show the case in which $n=2$ (and hence $s=\delta=1$ ). Then the case for general $n$ will follow by exactly the same argument as for $\beta \mathcal{P}^{q+s} \bar{\iota}_{2 q+\delta}$.

Because of the commutative diagram

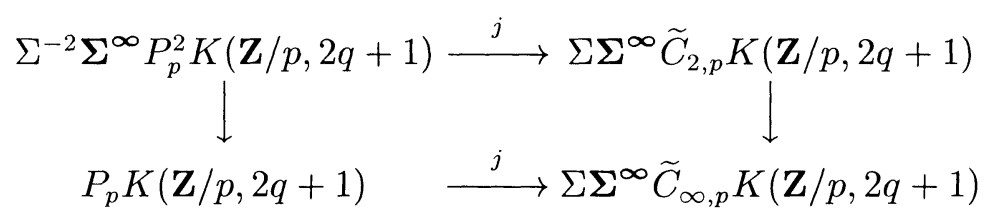

it suffices to show $\mathcal{P}^{q+1} \bar{\iota}_{2 q+1} \doteq j^{*}\left(\sigma \widetilde{Q}_{p-2} \iota_{2 q+1}\right)$ holds in $\widetilde{H}^{*}\left(P_{p} K(\mathbf{Z} / p, 2 q+1)\right)$. The class $\bar{\iota}_{2 q+1} \in \widetilde{H}^{2 q+1}\left(P_{p} K(\mathbf{Z} / p, 2 q+1)\right)$ is the unique element for which $i^{*} \bar{\iota}_{2 q+1}=\iota_{2 q+1}$. Since we have computed that $\beta \mathcal{P}^{q+1} \bar{\iota}_{2 q+1} \neq 0$ in 
$\widetilde{H}^{*}\left(P_{p}^{2} K(\mathbf{Z} / p, 2 q+1)\right)$, the above diagram shows us that $\mathcal{P}^{q+1} \bar{\iota}_{2 q+1} \neq 0$. However, $i^{*}\left(\mathcal{P}^{q+1} \bar{\iota}_{2 q+1}\right)=\mathcal{P}^{q+1} \iota_{2 q+1}=0$ by the unstable condition; therefore, $0 \neq$ $\mathcal{P}^{q+1} \bar{\iota}_{2 q+1} \in \operatorname{im} j^{*}$. On the other hand, because the space $P_{p}^{1} K(\mathbf{Z} / p, 2 q+1)$ has L-S category 2 , all threefold cup products vanish in its cohomology, and hence the image of

$$
\mathcal{P}^{q+1} \bar{\iota}_{2 q+1}=\bar{\iota}_{2 q+1}^{p} \quad \text { in } \quad \widetilde{H}^{2 p(q+1)}\left(P_{p}^{1} K(\mathbf{Z} / p, 2 q+1)\right)
$$

must be zero.

In order to simplify notation, let $K=K(\mathbf{Z} / p, 2 q+1)$. Consider the following commutative diagram.

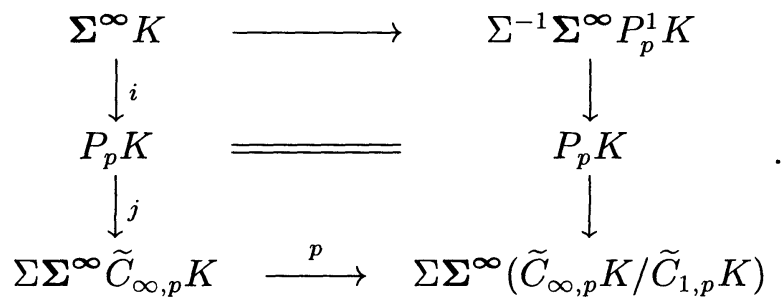

The discussion above implies that there is an element

$$
v \in \widetilde{H}^{2 p(q+1)-1}\left(\widetilde{C}_{\infty, p} K / \widetilde{C}_{1, p} K\right)
$$

for which $j^{*} p^{*}(v)=\mathcal{P}^{q+1} \bar{\iota}_{2 q+1} \neq 0$. Since all (external) Pontryagin product elements in the $\bmod p$ homology of $\widetilde{C}_{\infty, p} K$ must come from the $\bmod p$ homology of $\widetilde{C}_{1, p} K, p^{*}(v)$ must be equal (up to a unit in $\mathbf{F}_{p}$ ) to $\widetilde{Q}_{p-2} \iota_{2 q+1}$, since it is the only element in that degree which is in the image of $p^{*}$. Thus $\mathcal{P}^{q+1} \bar{\iota}_{2 q+1} \doteq j^{*}\left(\widetilde{Q}_{p-2} \iota_{2 q+1}\right)$ as desired.

For the general case, let $f: X \rightarrow K(\mathbf{Z} / p, 2 q+\delta)$ represent $x$. Then Theorem 6.1 of [KSW] implies that, for every choice of element $\bar{x}$ such that $i^{*}(\bar{x})=\sigma^{n} x$, there is a corresponding (unique) $H_{p}^{n}$-structure on $f$ (note that if $i^{*}(\bar{x})=0$, then $\bar{x} \in \operatorname{im} j^{*}$, and $\mathcal{P}^{q+s} \bar{x}=0$ by the unstable condition). Now consider the following commutative diagram.

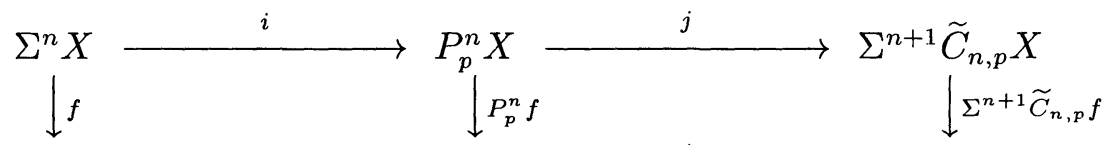

$\Sigma^{n} K(\mathbf{Z} / p, 2 q+\delta) \stackrel{i}{\longrightarrow} P_{p}^{n} K(\mathbf{Z} / p, 2 q+\delta) \stackrel{j}{\longrightarrow} \Sigma^{n+1} \widetilde{C}_{n, p} K(\mathbf{Z} / p, 2 q+\delta)$

By construction, $f^{*}\left(\iota_{2 q+\delta}\right)=x,\left(P_{p}^{n} f\right)^{*}\left(\bar{\iota}_{2 q+\delta}\right)=\bar{x}$ and $\left(\widetilde{C}_{n, p} f\right)^{*}\left(\widetilde{Q}_{r} \iota_{2 q+\delta}\right) \cdot=$ $\widetilde{Q}_{r} x$, and the result follows. QED

The following corollary is the stable version of Theorem 4.1. Recall from $[\mathbf{K S W}]$ that there is a natural map $\Sigma^{-n} \boldsymbol{\Sigma}^{\infty} P_{p}^{n} X \rightarrow P_{p} X$ for every 
$X \in \mathrm{Ob} \mathcal{H}_{p}^{\infty}$. The geometric filtration determined by these maps yields an algebraic filtration of $\widetilde{H}^{*}\left(P_{p} X\right)$, and we define

$$
F^{k+1}=\operatorname{ker}\left\{\widetilde{H}^{*}\left(P_{p} X\right) \rightarrow \widetilde{H}^{*}\left(\Sigma^{-k} \boldsymbol{\Sigma}^{\infty} P_{p}^{k} X\right)\right\} .
$$

Corollary 4.2. Suppose $p$ is an odd prime and $X \in \mathrm{Ob}^{\infty}$, with basic cofibration sequence

$$
\boldsymbol{\Sigma}^{\infty} \widetilde{C}_{\infty, p} X \stackrel{h}{\longrightarrow} \boldsymbol{\Sigma}^{\infty} X \stackrel{i}{\longrightarrow} P_{p} X \stackrel{j}{\longrightarrow} \Sigma \boldsymbol{\Sigma}^{\infty} \widetilde{C}_{\infty, p} X
$$

Let $n=2 s+1-\delta$ for some $\delta \in\{0,1\}$ and $s \geq \delta$. If $\bar{x} \in \widetilde{H}^{2 q+\delta}\left(P_{p} X\right)$, and $s>0$, then

$$
\begin{aligned}
\mathcal{P}^{q+s} \bar{x} & \doteq j^{*}\left(\sigma^{\infty} \widetilde{Q}_{(n-1)(p-1)-1} x\right)\left(\bmod F^{n+1}\right), \\
\beta \mathcal{P}^{q+s} \bar{x} & \doteq j^{*}\left(\sigma^{\infty} \widetilde{Q}_{(n-1)(p-1)} x\right)\left(\bmod F^{n+1}\right),
\end{aligned}
$$

where $i^{*} \bar{x}=\sigma^{\infty} x$ defines $x \in \widetilde{H}^{2 q+\delta}(X)$.

\section{Appendix: The Nishida relations.}

The Nishida relations $[\mathbf{N I}, \mathbf{C L M}]$, are formulas which relate the action of Dyer-Lashof operations to the (adjoint) action of the Steenrod algebra on $\widetilde{H}_{*}\left(\widetilde{C}_{n, p} X\right)$. In computations using the main theorem it is helpful to have the analogous formulas in cohomology. They are given in the next proposition.

Recall from Section 3 that $\widetilde{H}_{*}\left(\widetilde{C}_{n, p} X\right) \cong B \oplus C$, for certain submodules $B$ and $C$. Let $\operatorname{Ann}(B) \subset \widetilde{H}^{*}\left(\widetilde{C}_{n, p} X\right)$ be the submodule of elements which pair trivially with every element of $B$ under the Kronecker pairing.

Let $\delta \in\{0,1\}$ and define a function $\lambda$ by $\lambda(2 j+\delta)=\delta$. Finally, let $u \in \mathbf{F}_{p}$ denote an undetermined unit.

Proposition A.1. The following formulas (where (i) is taken mod $\operatorname{Ann}(B)$ in the case that $r=0$ ) hold for every $x \in \widetilde{H}^{q}(X)$.

(i)

$$
\begin{aligned}
& \mathcal{P}^{k} \widetilde{Q}_{r} x \doteq \sum_{i=0}^{[k / p]}\left(\begin{array}{c}
{[r / 2]+(p-1)(q / 2-i)} \\
k-p i
\end{array}\right) \widetilde{Q}_{r+2(p-1)(k-p i)} \mathcal{P}^{\imath} x \\
& +u \lambda(r-1) \sum_{i=0}^{[k / p]}\left(\begin{array}{c}
{[r / 2]+(p-1)(q / 2-i)-1} \\
k-p i-1
\end{array}\right) \widetilde{Q}_{r+2(p-1)(k-p i)-p} \beta \mathcal{P}^{i} x .
\end{aligned}
$$

(ii)

$$
\beta \widetilde{Q}_{2 r-1} x \doteq \widetilde{Q}_{2 r} x .
$$


Proof. It suffices to prove the formulas in $\widetilde{H}^{*}\left(D_{p} K(\mathbf{Z} / p, q)\right)$, with $x=\iota_{q}$, where they are dual to the usual Nishida formulas.

\section{References}

[BT] W. Browder and E. Thomas, On the projective plane of an $\mathrm{H}$-space, Ill. J. Math., 7 (1963), 493-501.

[BMMS] R. Bruner, J.P. May, J. McClure, and M. Steinberger, $H_{\infty}$ Ring Spectra and their Applications, Springer Verlag, LNM, 1176 (1986).

[CLM] F.R. Cohen, T.J. Lada, and J.P. May, The Homology of Iterated Loop Spaces, Springer Verlag, LNM, 533 (1976).

[CPS] H. Campbell, F. Peterson, and P. Selick, Self-Maps of Loop Spaces, I, Trans. Amer. Math. Soc., 293 (1986), 1-39.

[KSW] N.J. Kuhn, M. Slack, and F. Williams, A Hopf type construction for iterated loop spaces, preprint.

[Ma2] J.P. May, The Geometry of Iterated Loop Spaces, Springer Verlag, LNM 271 (1970).

[Mi] R.J. Milgram, Iterated loop spaces, Ann. Math., 11 (1967), 242-250.

[Ni] G. Nishida, Cohomology operations in iterated loop spaces, Proc. J. Acad., 44 (1968), 104-109.

[Sl] M. Slack, Infinite loop spaces with trivial Dyer-Lashof operations, Math. Proc. Camb. Phil. Soc., 113 (1993), 311-328.

[Th] E. Thomas, On functional cup-products and the transgression operator, Arch. Math., 12 (1961), 435-444.

[Za] A. Zabrodsky, Secondary operations in the cohomology of $H$-spaces, Illinois J. Math., 15 (1971), 648-655.

Received September 27, 1993.

JACKSONVILLE UNIVERSITY

JACKSONVILLE, FL 32211

E-mail address: mfoskey@junix.ju.edu

AND

Western Michigan University

Kalamazoo, MI 49008

E-mail address: slack@math-stat.wmich.edu 


\title{
PACIFIC JOURNAL OF MATHEMATICS
}

Founded by

\author{
$\begin{array}{ll}\text { E. F. Beckenbach (1906-1982) F. Wolf (1904-1989) } & \text { F }\end{array}$
}

EDITORS

Sun-Yung A. Chang (Managing Editor) Robert Finn

University of California

Los Angeles, CA 90095-1555

pacific@math.ucla.edu

F. Michael Christ

University of California

Los Angeles, CA 90095-1555

christ@math.ucla.edu

Nicholas Ercolani

University of Arizona

Tucson, AZ 85721

ercolani@math.arizona.edu
Stanford University

Stanford, CA 94305

finn@gauss.stanford.edu

Steven Kerckhoff

Stanford University

Stanford, CA 94305

spk@gauss.stanford.edu

Martin Scharlemann

University of California

Santa Barbara, CA 93106

mgscharl@math.ucsb.edu
Gang Tian

Massachusettes Institute of Technology

Cambridge, MA 02139

tian@math.mit.edu

\section{S. Varadarajan}

University of California

Los Angeles, CA 90095-1555

vsv@math.ucla.edu

Dan Voiculescu

University of California

Berkeley, CA 94720

dvv@math.berkeley.edu

\section{SUPPORTING INSTITUTIONS}

\author{
CALIFORNIA INSTITUTE OF TECHNOLOGY \\ NEW MEXICO STATE UNIVERSITY \\ OREGON STATE UNIVERSITY \\ STANFORD UNIVERSITY \\ UNIVERSITY OF ARIZONA \\ UNIVERSITY OF BRITISH COLUMBIA \\ UNIVERSITY OF CALIFORNIA \\ UNIVERSITY OF HAWAII
}

\author{
UNIVERSITY OF MONTANA \\ UNIVERSITY OF NEVADA, RENO \\ UNIVERSITY OF OREGON \\ UNIVERSITY OF SOUTHERN CALIFORNIA \\ UNIVERSITY OF UTAH \\ UNIVERSITY OF WASHINGTON \\ WASHINGTON STATE UNIVERSITY
}

The supporting Institutions listed above contribute to the cost of publication of this Journal, but they are not owners or publishers and have no responsibility for its contents or policies.

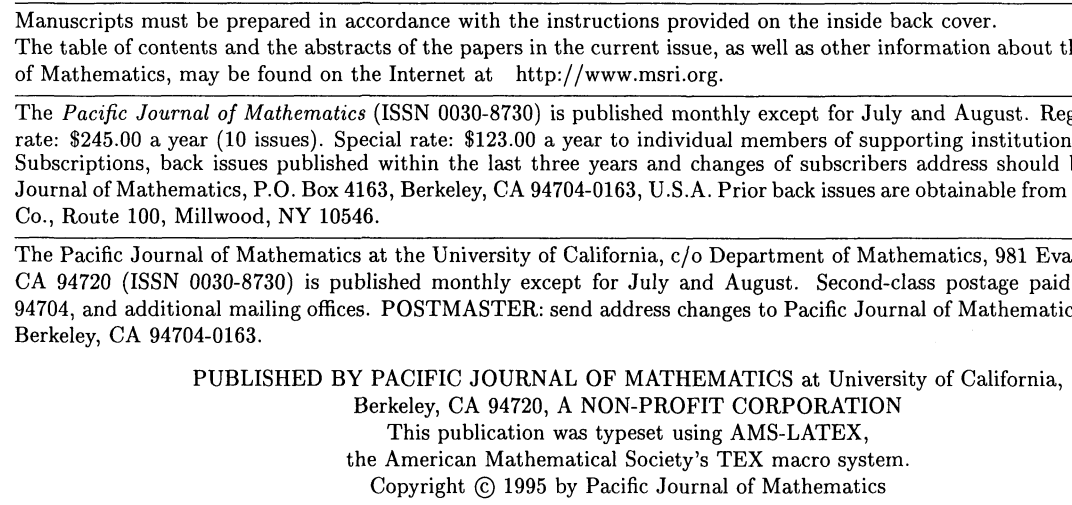




\section{PACIFIC JOURNAL OF MATHEMATICS}

\section{Volume $173 \quad$ No. $1 \quad$ March 1996}

Isometric immersions of $H_{1}^{n}$ into $H_{1}^{n+1}$

KINETSU ABE

Rotationally symmetric hypersurfaces with prescribed mean curvature

MARIE-FRANÇOISE BIDAUT-VÉRON

The covers of a Noetherian module

JIAN-JUN CHUAI

On the odd primary cohomology of higher projective planes

MARK FoSKEY and MiCHAEL DAVID SLACK

Unit indices of some imaginary composite quadratic fields. II

MiKinito HiRABAYASHI

Mixed automorphic vector bundles on Shimura varieties

MIN Ho LeE

Trace ideal criteria for Toeplitz and Hankel operators on the weighted Bergman

spaces with exponential type weights

PENG LIN and RICHARD ROCHBERG

On quadratic reciprocity over function fields

KATHY DONOVAN MERRILL and LYNNE WALLING

$\left(A_{2}\right)$-conditions and Carleson inequalities in Bergman spaces

TAKAHIKO NAKAZI and MASAHIRO YAMADA

A note on a paper of E. Boasso and A. Larotonda: "A spectral theory for solvable Lie 173 algebras of operators"
C. Отт

Tensor products with anisotropic principal series representations of free groups

Carlo Pensavalle and Tim Steger

On Ricci deformation of a Riemannian metric on manifold with boundary

YING SHEN

The Weyl quantization of Poisson $S U(2)$

Albert Jeu-Liang Sheu

Weyl's law for $S L(3, \mathbb{Z}) \backslash S L(3, \mathbb{R}) / S O(3, \mathbb{R})$

ERIC GeORge Stade and Dorothy IRENe Wallace (ANDREOli)

Minimal hyperspheres in two-point homogeneous spaces

PER TOMTER

Subalgebras of little Lipschitz algebras

NiKOLAI ISAAC WEAVER 